\title{
Real-World Experience of Treatment With Thrombopoietin Receptor Agonists in Anti-thymocyte Globulin-Naïve Patients With Aplastic Anemia: An Observational Retrospective Analysis in a Single Institution
}

\author{
Masaki lino ( $\square$ iinomasaki23@yahoo.co.jp ) \\ Tomoya Sato \\ Yamanashi Prefectural Central Hospital \\ Atsushi Jinguji \\ Yamanashi Prefectural Central Hospital \\ Ayato Nakadate \\ Yamanashi Prefectural Central Hospital
}

Yamanashi Prefectural Central Hospital https://orcid.org/0000-0002-3471-0890

\section{Research Article}

Keywords: aplastic anemia, thrombopoietin receptor agonist, eltrombopag, romiplostim, anti-thymocyte globulin.

Posted Date: October 25th, 2021

DOl: https://doi.org/10.21203/rs.3.rs-953779/v1

License: (c) (1) This work is licensed under a Creative Commons Attribution 4.0 International License. Read Full License

Version of Record: A version of this preprint was published at Hematology on March 20th, 2022. See the published version at https://doi.org/10.1080/16078454.2022.2045725. 


\section{Abstract}

Thrombopoietin receptor agonists (TPO-RAs) are used to treat bone marrow failure in aplastic anemia (AA). Treatment with TPO-RAs, cyclosporine A (CsA) and anti-thymocyte globulin (ATG) induces remission and a sustained response. However, the efficacy of TPO-RAs without ATG remains unclear. We retrospectively assessed 45 patients with AA refractory to CsA and naïve for ATG treatment who received eltrombopag without ATG at our hospital during 2017-2021. Of these, 28 (62\%) achieved a hematologic response in at least one lineage after six months of treatment, and 38 (84\%) achieved best response at any point during the follow-up period. Four patients (25\%) achieved trilineage responses during the follow-up period. Five patients switched from eltrombopag to romiplostim because of adverse events or lack of efficacy. Two developed hematologic malignancies. Eltrombopag was effective even in elderly ATG-ineligible patients with severe AA. The two-year overall survival rate was $84.3 \%$, with a median follow-up of 26.3 months. Time from diagnosis to eltrombopag treatment initiation tended to affect the response $(p=0.0727)$, but no factors that significantly

predicted hematologic response were identified. In conclusion, patients who are ineligible for ATG treatment because of age, complications, or even severe AA should nevertheless be considered for TPO-RA treatment.

\section{Introduction}

Aplastic anemia (AA) is a disease characterized by pancytopenia and hypoplastic bone marrow caused by an autoimmune mechanism [1]. Immunosuppressive treatment with anti-thymocyte globulin (ATG) and cyclosporine A (CsA) had been the standard therapy for mild to severe AA (SAA) as a first-line treatment for patients who are not candidates for hematopoietic stem cell transplantation [1].

Recently, the thrombopoietin receptor agonists (TPO-RAs) eltrombopag and romiplostim have become available for the treatment of refractory AA [2,3]. The efficacy of eltrombopag with a combination of ATG and CsA for treating newly diagnosed AA has also been shown [4]. These TPO-RAs activate TPO receptors on not only megakaryocytes but also primitive hematopoietic stem and progenitor cells, and they enhance hematopoiesis [5]. In addition, recent studies have demonstrated that TPO-RAs effectively recover trilineage hematopoiesis, which in some patients is sustained even after TOP-RA discontinuation. Based on these findings, recent treatment guidelines suggest that a combination of TPO-RAs with immunosuppressive therapy should be considered as a standard of care for AA patients [6-8].

In real-world clinical practice, there are some patients for whom ATG is not appropriate treatment, for reasons such as age, comorbid infection, concomitant complications, the patients' preference, and allergic reaction to ATG. However, most data on the administration of TPO-RAs to AA patients has been limited to clinical trials, and limited evidence has been reported for TPO-RAs in CsA-refractory ATG-naïve patients [9-11]. As a result, the efficacy of TPO-RAs for such patients in the real world remains unclear and is of special interest. In the present study, we retrospectively evaluated the outcome of treatment with TPO-RAs, mainly eltrombopag, for patients who either did not require or were unable to receive ATG, and explored the factors that were predictive of TPO-RA treatment outcome to optimize the treatment.

\section{Patients And Methods}

\subsection{Research participants and study design}

We reviewed the medical records of consecutive patients who had been diagnosed with AA between September 2017 and April 2021 at Yamanashi Prefectural Central Hospital. We retrospectively evaluated 45 patients with AA aged $\geq 16$ years who had been treated with TPO-RAs without ATG. We investigated the following patient characteristics: age, sex, severity of AA, complete blood count, duration from diagnosis to the initiation of TPO-RA treatment, bone marrow examination (including cytogenetic analysis at the initiation of TPO-RA treatment), positivity for paroxysmal nocturnal 
hemoglobinuria $(\mathrm{PNH})$ clone, transfusion dependency, number of prior treatments, type of prior treatments, type of TPORAs, maximal and maintenance dose of TPO-RAs, concomitant therapies during TPO-RA treatment, duration of TPO-RA treatment, hematologic response to TPO-RA treatment, adverse events during TPO-RA treatment, time to response, and overall survival. The TPO-RA eltrombopag was initially administered, in accordance with the approved indications for patients with AA in Japan $[8,11,12]$.

If the eltrombopag was not effective or resulted in serious adverse events, it was changed to romiplostim at the discretion of the treating physicians. In brief, eltrombopag was orally administered at a starting dose of $25 \mathrm{mg} / \mathrm{day}$; after two weeks of administration, dose adjustments in increments of $25 \mathrm{mg} /$ day were considered, up to a maximum dose of $100 \mathrm{mg} /$ day. Romiplostim was subcutaneously injected at a starting dose of $10 \mu \mathrm{g} / \mathrm{kg}$ once per week; this was adjusted in increments of $5 \mu \mathrm{g} / \mathrm{kg}$ up to a maximum of $20 \mu \mathrm{g} / \mathrm{kg}$, according to hematopoietic recovery [13]. After a stable trilineage response had been achieved, the TPO-RA dosage was decreased gradually and then discontinued, while trilineage hematopoiesis was sustained. TPO-RA treatment was resumed when a loss of the response was observed, at the discretion of the treating physicians. Cytogenetic analysis (G-band staining and fluorescence in situ hybridization [FISH]) for chromosome 7q deletion was performed every 6-12 months or when a loss of treatment response was observed, using bone marrow or peripheral blood samples.

This study was approved by the ethics committee of Yamanashi Prefectural Central Hospital and was conducted in accordance with the tenets of the Declaration of Helsinki. All patients provided written informed consent.

\subsection{Definitions}

AA and the levels of disease severity have been defined previously [6-8]. Based on previous research [11], a platelet response was defined as an increase in platelet count of $\geq 20 \times 10^{9} / \mathrm{L}$ above the baseline, or independence from platelet transfusions for at least eight weeks in patients who were previously transfusion dependent. An erythroid response in patients with a pretreatment hemoglobin level of $<9 \mathrm{~g} / \mathrm{dL}$ was defined as an increase in the hemoglobin level by $\geq 1.5$ $\mathrm{g} / \mathrm{dL}$ without transfusion, or a reduction of at least four transfusion units for eight weeks relative to transfusion requirements during the eight weeks immediately preceding the first administration of the TPO-RA. A neutrophil response was defined as an increase in the absolute neutrophil count (ANC) of $>0.5 \times 10^{9} / \mathrm{L}$, or at least a doubling of ANC from baseline in patients with a baseline ANC of $\leq 0.5 \times 10^{9} / \mathrm{L}$. In patients who had an ANC $\geq 1.0 \times 10^{9} / \mathrm{L}$ and/or a platelet count $\geq 100 \times 10^{9} / \mathrm{L}$ at study entry, increases in ANC and/or platelet count were not considered for response assessment. Time to hematologic response was defined as the duration from the initiation of eltrombopag administration to any hematologic response. Overall survival was defined as the duration from the first day of eltrombopag administration to death from any cause. Adverse events during TPO-RA treatment were evaluated in accordance with the National Cancer Institute's Common Terminology Criteria, version 5.0 [14].

\subsection{Statistical analysis}

Categorical and continuous variables were analyzed using Fisher's exact tests and t-tests, respectively. Differences with a p-value of $<0.05$ were considered statistically significant. Overall survival (OS) was estimated using the Kaplan-Meier method [15]. All statistical analyses were performed using EZR computer software [16].

\section{Results}

\subsection{Patient characteristics}

Forty-five patients with AA were treated with eltrombopag during the study period; their characteristics are summarized in Table 1. The median age was 76.5 years (range: $44-88$ years). Of these 45 patients, 10 had SAA, while the remaining 
35 had non-severe AA. The median reticulocyte count, hemoglobin level, ANC, and platelet count at the initiation of eltrombopag administration were 41,580 × 106/L (range: 7,840-80,860 × 106); $8.6 \mathrm{~g} / \mathrm{dL}$ (range: 5.0-14.8); $925 \times 10^{6} / \mathrm{L}$ (range: $120-6,622 \times 10^{6}$ ); and $16 \times 10^{9} / \mathrm{L}$ (range: $2-109 \times 10^{9}$ ), respectively. The median time from the diagnosis of AA to the initiation of eltrombopag administration was 5.7 months (range: 2.2-18.3). Four patients had an abnormal karyotype (trisomy 8 [ $n=1]$, 13q deletion [ $=1$ ], and loss of $Y$ chromosome [n = 2]), and 38 had a normal karyotype at the initiation of eltrombopag administration. PNH clone $(\geq 1 \%)$ was detected in eight patients $(18 \%)$. The median number of prior treatments was one (range: 1-2). CsA was the most common prior medicine administered to all patients, with a median duration of 4.1 months. Thirty-eight patients had failed to respond to previous treatment with CsA with metenolone, five had failed to respond to CsA alone, and the remaining two had failed to respond to CsA with prednisolone. Of the 45 patients, 43 were eligible for response assessment using the platelet lineage (platelets $<100 \times 10^{3} / \mu \mathrm{L}$ or dependent on platelet transfusions), 29 were eligible for response assessment using the erythroid lineage ( $\mathrm{Hb}<9 \mathrm{~g} / \mathrm{dL}$ or dependent on red blood cell transfusions), and 29 were eligible for response assessment using the neutrophil lineage (ANC $<1.0 \times$ $10^{9} / \mathrm{L}$ ). The reasons for avoiding treatment with ATG were as follows: advanced age ( $\geq 70$ years), $n=22 ;$ not recommended according to the Japanese guidelines $[8]^{101}, n=13$; patient preference, $n=6$; uncontrolled heart failure, $n$ $=2$; uncontrolled diabetes mellitus, $n=2$; chronic renal failure, $n=2$; invasive aspergillus infection, $n=1$.

\subsection{Clinical outcomes}

Initially, all patients received eltrombopag when the TPO-RA treatment commenced, because eltrombopag is a convenient oral tablet and was approved earlier in Japan than romiplostim. However, five patients needed to switch to romiplostim because of adverse events (anorexia and nausea grade $2, n=3$ ) or lack of efficacy $(n=2)$. The median duration of TOP-RA administration was 23.4 months (range: 2.1-48.3). The median maximum dose of eltrombopag or romiplostim was $100 \mathrm{mg} /$ day (range: 50-100) or $20 \mu \mathrm{g} / \mathrm{kg}$ (range: 15-20), respectively. CsA was the main concomitant medicine administered to 43 patients, with a median duration of 6.3 months. Metenolone was the second most common concomitant medicine. The change in eltrombopag dose over time is shown in Figure 1. The median eltrombopag maintenance dose for responders (in at least one lineage) and non-responders was $75 \mathrm{mg} /$ day (range: 12.5-100) and 50 $\mathrm{mg} /$ day (range: $25-100)$, respectively $(p=0.19)$. The median eltrombopag administration duration in responders and non-responders was 4.3 months (range: $2.1-8.4$ ) and 26.5 months (range: $3.4-48.3$ ), respectively $(p=0.028)$. Two patients received granulocyte colony-stimulating factor (G-CSF) for periods of infection; however, no neutrophil response was observed in these patients.

\subsection{Hematologic improvements resulting from TPO-RA treatment in patients with AA}

Of these 45 patients, a response was observed in at least one lineage in 38, with a median time to response of 84 days, and trilineage responses were observed in four patients, with median time to response of 397 days (Tables 2 and 3 , Figure 2). Hematologic responses over time are shown in Figure 3. Of the 10 transfusion-dependent patients, two became transfusion-independent and the requirement for reduced transfusions reduced in two. However, one male patient became transfusion-dependent after the onset of acute myeloid leukemia (AML). All patients who had a hematologic response, except those who died or progressed to AML or myelodysplastic syndrome (MDS), were continuing to be administered with TPO-RAs at the time of analysis.

\subsection{Adverse events and evolution to myeloid malignancy}

Adverse events were observed in 18 of the 45 patients (40\%, Table 4) during the TPO-RA treatment. Common adverse events were anorexia $(n=4)$, nausea $(n=4)$, lung infection $(n=3)$, oral submucosal hemorrhage $(n=2)$, blood liver transaminase elevation $(n=2)$, blood bilirubin elevation $(n=2)$, headache $(n=2)$, and febrile neutropenia $(n=2)$; however, most of these were not serious. Regarding serious adverse events, liver dysfunction was observed in a 44-year- 
old patient who was receiving concomitant eltrombopag with CsA. He developed grade 2 upper abdominal pain and liver dysfunction, with elevation of serum transaminase and hyperbilirubinemia (grade 3 blood aspartate and alanine aminotransferase [ALT and AST] increase and grade 1 blood bilirubin increase) after two months of administration of eltrombopag at $75 \mathrm{mg} /$ day. After discontinuation of eltrombopag for 22 days, his symptoms and liver function were resolved, and eltrombopag administration was then restarted at a dose of $25 \mathrm{mg} /$ day, which was then increased to the maintenance dose (100 mg/day) and maintained until the time of analysis. A 52-year-old woman developed grade 2 anorexia, grade 2 nausea, grade 1 vomiting and liver dysfunction (grade 2 AST and ALT increase and grade 2 hyperbilirubinemia) after four months of administration with eltrombopag at $100 \mathrm{mg} / \mathrm{day}$ with concomitant CsA. After discontinuation of eltrombopag for 20 days, her appetite and liver function recovered, and eltrombopag treatment was resumed at a dose of $25 \mathrm{mg} /$ day. However, when the dose was increased to $75 \mathrm{mg} /$ day, the anorexia and nausea returned, and at that stage the eltrombopag was switched to romiplostim. Two other women, aged 77 and 76 , both experienced grade 2 anorexia and grade 2 nausea, and their eltrombopag treatment was also switched to romiplostim. Eventually, these three women were receiving the maximum dose of romiplostim subcutaneously once a week, and this resulted in uni- to trilineage recovery. After switching to romiplostim, these three women had not again experienced the adverse events that they experienced prior to switching to romiplostim, as at the time of analysis.

The two-year OS rate was $84.3 \%$ (95\% confidence interval: 67.9-92.7\%), and five deaths were recorded, with a median follow-up period of 26.3 months (range: 2.1-45.3). The deaths were caused by infections (worsening invasive aspergillus pneumonia $[n=1]$, pyelonephritis $[n=1]$, and aspiration pneumonia $[n=1])$ and myeloid malignancy $(n=2)$.

None of the patients had cytogenetic abnormalities in chromosome 7 detected via FISH during the follow-up period. However, two patients who had a normal karyotype at the initiation of eltrombopag administration developed myeloid malignancies. A 67-year-old man achieved a unilineage response (platelets) after two months of eltrombopag administration. However, his platelet count started declining again after 11 months of treatment. Bone marrow examination revealed that he had hypoplastic AML with a blast percentage of $28 \%$. Low-dose cytarabine and anthracycline were not effective, and he died 20 months after the initiation of eltrombopag treatment. The other patient, a 66-year-old man, achieved a trilineage response after a month of eltrombopag administration. However, he started to develop pancytopenia after six months of treatment. The eltrombopag was switched to romiplostim, but this did not improve the pancytopenia. Bone marrow examination revealed that he suffered from MDS with multilineage dysplasia. Azacytidine was administered, but it was not effective, and he died 12 months after the initiation of eltrombopag treatment.

\subsection{Factors affecting hematologic improvements after initiation of TPO-RA administration in patients with ATG-naïve AA}

In univariate analysis, we investigated the following factors that may affect the response at six months: age, sex, severity of $\mathrm{AA}$, positivity for $\mathrm{PNH}$ clone, $\mathrm{ANC}$, reticulocyte, platelet counts at the initiation of eltrombopag administration, maintenance dose of eltrombopag and time from diagnosis to eltrombopag treatment initiation (Table 5). The time from diagnosis to treatment initiation tended to influence the response in at least one lineage, but this was not significant $(p=$ 0.0727 , Table 3). Neither univariate nor multivariate analysis revealed any factors that were predictive of the response.

\section{Discussion}

In this study, we have demonstrated the efficacy of TPA-RAs without ATG for patients with AA in a real-world setting. The median age of the patients in this cohort was 76.5 years old, which is higher than that in previous prospective studies (32-53 years) [2-4, 11, 17-19], although in the one retrospective study we reviewed [9], the median age of patients naïve for ATG treatment was 74 years. The cohort in this study may therefore be more representative of the patients who 
would typically be naïve for ATG treatment in daily clinical practice. Of the 45 patients included in this study, 32 were ineligible for ATG treatment because of their age ( $\geq 70$ years) and/or comorbidities, and the other 13 had only mild symptoms and were not indicated for ATG treatment according to the Japanese guideline [8]. Hematologic response in at least one lineage was observed in $62 \%$ of patients at six months from the initiation of eltrombopag treatment, and an optimal response was observed in $84 \%$ of patients regardless of time. A trilineage response was observed in $25 \%$ of eligible patients at any point during the follow-up period. The median time to achieve hematological responses in at least one lineage and a trilineage response were 84 and 397 days, respectively.

Several prospective and retrospective studies have published data on the efficacy and safety of TPO-RA for newly diagnosed and refractory $A A[2-4,9,11,17-19]$. In these studies, the response rates for at least unilineage and trilineage responses were approximately $40-80 \%$ and $20-40 \%$, respectively.

In these studies, most patients had previously been treated with ATG, and one of these studies demonstrated that patients previously treated with ATG exhibited faster hematopoietic cell recovery (transfusion independence rate at three months: $44 \%$ versus $0 \%$ ) and a slightly better hematological response rate than patients not treated with ATG (response rate in at least one lineage: $74 \%$ vs $64 \%$; trilineage response rate: $34 \%$ vs $27 \%$ ) [9]. Although the numbers of patients are small and there are obvious caveats concerning attempts to compare independent studies, the hematologic response rates and speed of hematopoietic recovery in our study do not seem to be inferior to the results of previous studies, including those involving younger patients and those previously treated with ATG.

This study cohort included 10 patients with SAA. Of these, six were over 75 years old, and the response rate for at least unilineage and trilineage responses in this group was $66 \%$ and $33 \%$, respectively. Immunosuppressive therapy is effective regardless of the patient's age; however, the mortality rate is likely to be higher in older patients than younger patients $[20,21]$. Therefore, clinicians should take extra care when administering ATG to elderly patients. However, previous case reports have demonstrated the efficacy of eltrombopag without ATG for treating SAA [22, 23], and findings in our study were consistent with these reports even in elderly patients who are ineligible for ATG treatment. These findings suggest that TPO-RA should be administered even to elderly SAA patients who are ineligible for ATG.

The safety profile of the eltrombopag treatment in this study was consistent with previous studies $[2,4,11]$. The most frequent adverse event was anorexia and nausea. These events were resolved within two to four weeks of discontinuation or reduction of eltrombopag administration. Although the adverse events did not recur in most of the patients when the eltrombopag was reinitiated, they did in three female patients, who were then switched to romiplostim. After the switch, their symptoms were completely resolved. Most of the adverse events were not serious and appear not to have been related to age. Instead, anorexia and nausea were more likely to be observed in female patients. Because the number of patients in this study was small, however, further data collection will be necessary to confirm these tendencies.

By the time of data analysis, five patients had been switched from eltrombopag to romiplostim. The reason for this in the other two patients (who were male) was lack of efficacy. After switching to romiplostim, the response was improved in one of these patients, but not in the other, due to the onset of MDS. Previous studies have shown that switching from one TPO-RA to another is effective in clinical practice in patients with not only immune thrombocytopenia [24, 25] but also AA $[10,26]$. Our observational findings were consistent with their results. In this study, the eltrombopag dosage tended to affect the hematological response. An adequate TPO-RA dosage appears to be necessary for hematological recovery. If the first TPO-RA fails because an adequate dosage cannot be provided, switching to another TPO-RA should be considered to achieve treatment success.

Our study cohort included two patients who developed hematological malignancy. These patients eventually died as a result of $A M L$ and MDS, respectively. In previous studies on TPO-RA treatment for AA, clonal evolution and AML have 
been observed at an incidence of $3-19 \%$ and $0-4 \%$, respectively $[2,4,17,19,27]$. However, it is also reported that secondary MDS or AML occurs in 15-20\% of patients with AA regardless of the use of TPO-RAs [28]. TPO-RAs can stimulate the expansion of hematopoietic stem cells via the c-MPL signaling pathway [5] and this could potentially affect the emergence of abnormal clones. Clonal evolution is of concern, and the precise mechanism underlying the development of hematologic malignancy remains controversial. Therefore, long-term follow-up and close surveillance of patients treated with TPO-RAs are essential.

We did not find any factors that significantly predicted hematological response to eltrombopag treatment. A shorter period from AA diagnosis to treatment initiation was likely to predict a hematopoietic response, but it was not significant. Previous studies have shown that higher reticulocyte counts and a shorter period from first immunosuppressive therapy to initiation of eltrombopag administration were likely to predict a hematological response to eltrombopag $[2,27]$. While there are sufficient hematopoietic stem cells remaining in the bone marrow (i.e., before the hematopoietic stem cells are depleted), the introduction of a TPO-RA may be able to effectively stimulate a hematologic response.

This study has some limitations. First, it was a retrospective observational study with a small number of patients being treated at a single institution. The decisions to switch from pretreatment to TPO-RA when the TPO-RA was introduced and to switch the TPO-RA from eltrombopag to romiplostim were therefore left to the discretion of the treating physicians. To confirm our findings, we need to conduct a prospective study with a larger number of patients. Second, the reasons for not using ATG involved both cases in which the symptoms were mild and the use of ATG was unnecessary (i.e., not recommended according to the guidelines), and cases in which the symptoms were severe but ATG could not be used because of patient factors such as age and complications. There is thus a need for studies involving sufficient numbers of cases that the patients can be divided into separate cohorts according to these two sets of characteristics. Third, clonal evolution was not evaluated systematically, and it might occur more frequently than was documented here.

In conclusion, we investigated the efficacy of TPA-RAs in ATG- naïve patients with AA. Our findings reflect the nature of real-world TOP-RA treatment in patients with AA and suggest that TPO-RAs should be attempted in refractory patients with AA, even if they are elderly, have severe AA, and are ATG-ineligible. Further studies may identify factors that predict efficacy. Better understanding of TPO-RA action in AA and the proper use of TPO-RA in AA patients could improve AA treatment strategies and encourage successful long-term treatment of patients with ATG- naïve AA.

\section{Declarations}

Acknowledgments: We would like to all the patients who participated in this study. We would like to thank Uni-edit (https://uni-edit.net/) for editing and proofreading this manuscript.

Conflicts of interest: The authors declare that they have no conflicts of interest.

Authors' contributions: MI designed the study and wrote the initial draft of the manuscript. MI, TS, AJ and AN contributed to data collection and interpretation and critically reviewed the manuscript. MI, TS, AJ and AN approved the final version of the manuscript and agree to be accountable for all aspects of the work in ensuring that questions related to the accuracy or integrity of any part of the work are appropriately investigated and resolved.

\section{References}

1. Young NS. Aplastic anemia. N Engl J Med. 2018;379:1643-56. 
2. Olnes MJ. Scheinberg P, Calvo KR. Desmond R, Tang Y. Dumitriu B, et al. Eltrombopag and improved hematopoiesis in refractory aplastic anemia. N Engl J Med. 2012;367:11-9.

3. Lee JW. Lee S, Jung CW. Park S, Keta H. Park SK, et al. Romiplostim in patients with refractory aplastic anaemia previously treated with immunosuppressive therapy: a dose-finding and long-term treatment phase 2 trial. Lancet Haematol. 2019;6:e562-72.

4. Townsley DM, Scheinberg P, Winkler T, Desmond R, Dumitriu B, Rios O, et al. Eltrombopag added to standard immunosuppression for aplastic anemia. N Engl J Med. 2017;376:1540-50.

5. Zeigler BFC, Sauvage F de, Widmer HR, Keller GA, Donahue C, Schreiber RD, et al. In vitro megakaryocytopoietic and thrombopoietic activity of c-mpl ligand (TPO) on purified murine hematopoietic stem cells. Blood. 1994;84(12):4045-52.

6. Killick SB. Bown N, Cavenagh J. Dokal I, Foukaneli T. Hill A, et al. Guidelines for the diagnosis and management of adult aplastic anaemia. Br J Haematol. 2016;172:187-207.

7. Miano M. Dufour C. The diagnosis and treatment of aplastic anemia: a review. Int J Hematol. 2015;101:527-35.

8. Nakao S. Hama M, Ohashi H. Usuki K, Inokuchi K. Suzuki T, et al. Reference guide for the treatment of aplastic anemia. 2019 revised version. http://zoketsushogaihan.umin.jp/file/2020/02.pdf Accessed 2021 September $26 .$.

9. Lengline E. Drenou B, Peterlin P. Tournilhac O, Abraham J. Berceanu A, et al. Nationwide survey on the use of eltrombopag in patients with severe aplastic anemia: A report on behalf of the French reference center for aplastic anemia. Haematologica. 2018;103:212-20.

10. Ise M. lizuka H, Kamoda Y. Hirao M, Kida M. Usuki K. Romiplostim is effective for eltrombopag-refractory aplastic anemia: results of a retrospective study. Int J Hematol. 2020;112:787-94.

11. Yamazaki H. Ohta K, lida H. Imada K, Obara N. Tokumine Y. Hematologic recovery induced by eltrombopag in Japanese patients with aplastic anemia refractory or intolerant to immunosuppressive therapy. Int J Hematol. 2019;110:187-96.

12. REVOLADE Tablets prescribing information. 2018. https://www.info.pmda.go.jp/go/pack/3999028F1025_2_05/? view=frame\&style=SGML\&lang=ja Accessed 2021 September 26.

13. Romiplate for s.c. injection prescribing information. 2020. https://www.info.pmda.go.jp/go/pack/3999430D1024_1_07/?view=frame\&style=XML\&lang=ja Accessed 2021 September 26.

14. Common terminology criteria for adverse events (CTCAE) version 5.0 https://ctep.cancer.gov/protocoldevelopment/electronic_applications/docs/CTCAE_v5_Quick_Reference_8.5x11.pdf Accessed 2021 September 26.

15. Kaplan EL. Meier P. Nonparametric estimation from incomplete observations. Source J Am Stat Assoc. 1958;53:457-81.

16. Kanda Y. Investigation of the freely available easy-to-use software 'EZR' for medical statistics. Bone Marrow Transplant. 2013;48:452-8.

17. Desmond R. Townsley DM, Dumitriu B. Olnes MJ, Scheinberg P. Bevans M, et al. Eltrombopag restores trilineage hematopoiesis in refractory severe aplastic anemia that can be sustained on discontinuation of drug. Blood. 2014;123:1818-25.

18. Fan X. Desmond R, Winkler T. Young DJ, Dumitriu B. Townsley DM, et al. Eltrombopag for patients with moderate aplastic anemia or uni-lineage cytopenias. Blood Adv. 2020;4:1700-10.

19. Jang JH. Tomiyama Y, Miyazaki K. Nagafuji K, Usuki K. Uoshima N, et al. Efficacy and safety of romiplostim in refractory aplastic anaemia: a Phase II/III, multicentre, open-label study. Br J Haematol. 2021;192:190-9. 
20. Sasaki N. Shimura K, Yoshida M. Uoshima N, Kiyota M. Hatsuse M, et al. Immunosuppressive therapy with rabbit antithymocyte globulin therapy for acquired aplastic anemia: a multi-institutional retrospective study in Japanese adult patients. Int J Hematol. 2019;109:278-85.

21. Tichelli A. Socié G, Henry-Amar M. Marsh J, Passweg J. Schrezenmeier H, et al. Effectiveness of immunosuppressive therapy in older patients with aplastic anemia. Ann Intern Med. 1999;130:193-201.

22. Rodgers GM. Gilreath JA. Eltrombopag as initial monotherapy for severe aplastic anemia-a case report. Ann Hematol. 2018;97:1517-8.

23. Cheng $\mathrm{H}$. Wang $\mathrm{X}, \mathrm{Xu} \mathrm{KL}$. Eltrombopag combined with cyclosporine may have an effective on very severe aplastic anemia. Ann Hematol. 2019;98:2009-11.

24. González-Porras JR. Godeau B, Carpenedo M. Switching thrombopoietin receptor agonist treatments in patients with primary immune thrombocytopenia. Ther Adv Hematol. 2019;10:2040620719837906.

25. Kuter DJ. Macahilig C, Grotzinger KM. Poston SA, Wang PF. Dawson KL, et al. Treatment patterns and clinical outcomes in patients with chronic immune thrombocytopenia (ITP) switched to eltrombopag or romiplostim. Int $\mathrm{J}$ Hematol. 2015;101:255-63.

26. Hosokawa K. Yamazaki H, Tanabe M. Imi T, Sugimori N. Nakao S. High-dose romiplostim accelerates hematologic recovery in patients with aplastic anemia refractory to eltrombopag. Leukemia. 2021;35:906-9.

27. Winkler T. Fan X, Cooper J. Desmond R, Young DJ. Townsley DM, et al. Treatment optimization and genomic outcomes in refractory severe aplastic anemia treated with eltrombopag. Blood. 2019;133:2575-85.

28. Sun L. Babushok DV. Secondary myelodysplastic syndrome and leukemia in acquired aplastic anemia and paroxysmal nocturnal hemoglobinuria. Blood. 2020;136:36-49.

\section{Tables}

Table 1. Baseline patient characteristics 
SAAPatients

Patient age (years), median (range)

Sex (female/male), n (\%)

Severity at initiation of Epag, $\mathrm{n}(\%)$

SAA

NSAA

Reticulocyte count at initiation of Epag $\left(\times 10^{6} / \mathrm{L}\right)$, median (range)

Hemoglobin level at initiation of Epag $(\mathrm{g} / \mathrm{dL})$, median (range)

ANC at initiation of Epag $\left(\times 10^{6} / \mathrm{L}\right)$, median (range)

Platelet count at initiation of Epag $\left(\times 10^{9} / \mathrm{L}\right)$, median (range)

Time from diagnosis to initiation of Epag (months), median (range)

Karyotype

Normal

Abnormal

Unknown

PNH clone

Positive ( $\geq 1 \%)$

Negative $(<1 \%)$

Unknown

Transfusion dependency

Platelet

Red blood cell

Number of prior treatments, median (range)

Prior treatments, $\mathrm{n}(\%)$

Cyclosporine A

Metenolone acetate

Prednisolone

Eligible for platelet response assessment

(platelets $<10 \times 10^{9} / \mathrm{L}$ or transfusion dependent), n (\%)

Eligible for erythroid response assessment

(hemoglobin $<9 \mathrm{~g} / \mathrm{dL}$ or transfusion dependent), $\mathrm{n}(\%)$

Eligible for neutrophil response assessment $\left(<1 \times 10^{9} / \mathrm{L}\right), \mathrm{n}(\%)$
$29(64)$

$n=45$

$76.5(44-88)$

$27 / 18(60 / 40)$

$10(22)$

$35(78)$

$41,580(7,840-80,860)$

$8.6(5.0-14.8)$

$925(120-6,622)$

$16(2-109)$

$5.7(2.2-18.3)$

38 (84)

4 (9)

3 (7)

8 (18)

34 (75)

3 (7)

$5(11)$

7 (16)

$1(1-2)$

45 (100)

38 (84)

2 (4)

43 (96)

$29(64)$ 
Abbreviations: ANC, absolute neutrophil count; Epag, eltrombopag; NSAA, non-severe aplastic anemia; $\mathrm{PNH}$, paroxysmal nocturnal hemoglobinuria; SAA, severe aplastic anemia.

Table 2. Outcomes of TPO-RA treatments

Patients

$\mathrm{n}=45$

Type of TPO-RA

Epag alone, $\mathrm{n}(\%)$

$40(89)$

Epag followed by Rom, n (\%)

5 (11)

Maximum dose of TPO-RA

Epag (mg), median (range)

$100(50-100)$

Rom ( $\mu \mathrm{g} / \mathrm{kg})$, median (range)

$20(15-20)$

Maintenance dose of TPO-RA

Epag (mg), median (range)

$75(12.5-100)$

Rom ( $\mu \mathrm{g} / \mathrm{kg})$, median (range)

$20(15-20)$

Concomitant AA therapy

Cyclosporine A

$43(96)$

Metenolone acetate

$29(64)$

None

$6(13)$

Time to response

Time to unilineage response (days), median (range)

$84(6-1,027)$

Time to trilineage response (days), median (range)

$397(185-656)$

Abbreviations: AA, aplastic anemia; Epag, eltrombopag; Rom, romiplostim; TPO-RA, thrombopoietin receptor agonist.

Table 3. Hematologic response to TPO-RAs by lineage

Response

\begin{tabular}{llll}
\hline & & At 6 months & Entire period \\
\hline Any response & 45 & $28(62)$ & $38(84)$ \\
\hline Platelet response & 43 & $16(37)$ & $24(56)$ \\
\hline Erythroid response & 29 & $8(28)$ & $11(38)$ \\
\hline Neutrophil response & 29 & $15(52)$ & $22(76)$ \\
\hline Trilineage response & 16 & $2(13)$ & $4(25)$ \\
\hline
\end{tabular}

Abbreviations: TPO-RA, thrombopoietin receptor agonist. 
Table 4. Adverse events during TPO-RA treatment

\begin{tabular}{llll}
$\mathrm{n}=45$ & Any grade & Grade 3-4 & Grade 5 \\
\cline { 2 - 3 } & No. of patients $(\%)$ & \\
\hline All adverse events & $18(40)$ & $3(7)$ & $5(11)$ \\
\hline Anorexia & $4(9)$ & 0 & 0 \\
\hline Nausea & $4(9)$ & 0 & 0 \\
\hline Lung infection & $3(7)$ & 0 & $2(4)$ \\
\hline Oral submucosal hemorrhage & $2(4)$ & 0 & 0 \\
\hline Blood AST increased & $2(4)$ & $1(2)$ & 0 \\
\hline Blood ALT increased & $2(4)$ & $1(2)$ & 0 \\
\hline Blood bilirubin increased & $2(4)$ & 0 & 0 \\
\hline Headache & $2(4)$ & 0 & 0 \\
\hline Febrile neutropenia & $2(4)$ & $2(4)$ & 0 \\
\hline Urinary tract infection & $1(2)$ & 0 & $1(2)$ \\
\hline Leukemia & $1(2)$ & 0 & $1(2)$ \\
\hline Myelodysplastic syndrome & $1(2)$ & 0 & $1(2)$ \\
\hline Hyperglycemia & $1(2)$ & 0 & 0 \\
\hline Abdominal pain upper & $1(2)$ & 0 & 0 \\
\hline Hemorrhoidal hemorrhage & $1(2)$ & 0 & 0 \\
\hline Skin rash & $1(2)$ & 0 & 0 \\
\hline Vomiting & $1(2)$ & 0 & 0 \\
\hline Blood creatinine increased & & 0 & 0 \\
\hline Abbreviations: ALT, alanine aminotransferase; AST, aspartate aminotransferase; & & 0 \\
\hline TPO-RA, thrombopoietin receptor agonist. & & 0 \\
\hline
\end{tabular}

Table 5. Univariate analysis of factors predicting response to eltrombopag 


\begin{tabular}{lllll} 
Factors & & Patients, & Unilineage response & $p^{a}$ \\
\cline { 2 - 4 } & & $n$ & at 6 months $(\%)$ & 0.543 \\
\hline Age & $<75$ vs $\geq 75$ years old & 19 vs 26 & 68 vs 58 & 0.351 \\
\hline Sex & female vs male & 27 vs 18 & 56 vs 72 & 0.719 \\
\hline AA severitiy & non-severe vs severe & 35 vs 10 & 60 vs 70 & 0.406 \\
\hline PNH clone & $<1$ vs $\geq 1 \%$ & 34 vs 8 & 71 vs 50 & 1.000 \\
\hline ANC & $<1.0$ vs $\geq 1.0 \times 10^{9} / \mathrm{L}$ & 27 vs 18 & 63 vs 61 & 0.233 \\
\hline Reticulocyte count & $<40$ vs $\geq 40 \times 10^{9} / \mathrm{L}$ & 21 vs 24 & 52 vs 71 & 0.759 \\
\hline Platelet count & $<20$ vs $\geq 20 \times 10^{9} / \mathrm{L}$ & 28 vs 17 & 64 vs 59 & 0.216 \\
\hline Maintenance dose of Epag & $<75$ vs $\geq 75 \mathrm{mg} / \mathrm{day}$ & 18 vs 27 & 50 vs 69 & 0.0727 \\
\hline Time from AA diagnosis & $<6$ vs $\geq 6$ months & 24 vs 21 & 75 vs 48 & . \\
\hline
\end{tabular}

Abbreviations: AA, aplastic anemia; ANC, absolute neutrophil count; Epag, eltrombopag; $\mathrm{PNH}$, paroxysmal nocturnal hemoglobinuria.

${ }^{a}$ Fisher's exact test

\section{Figures}




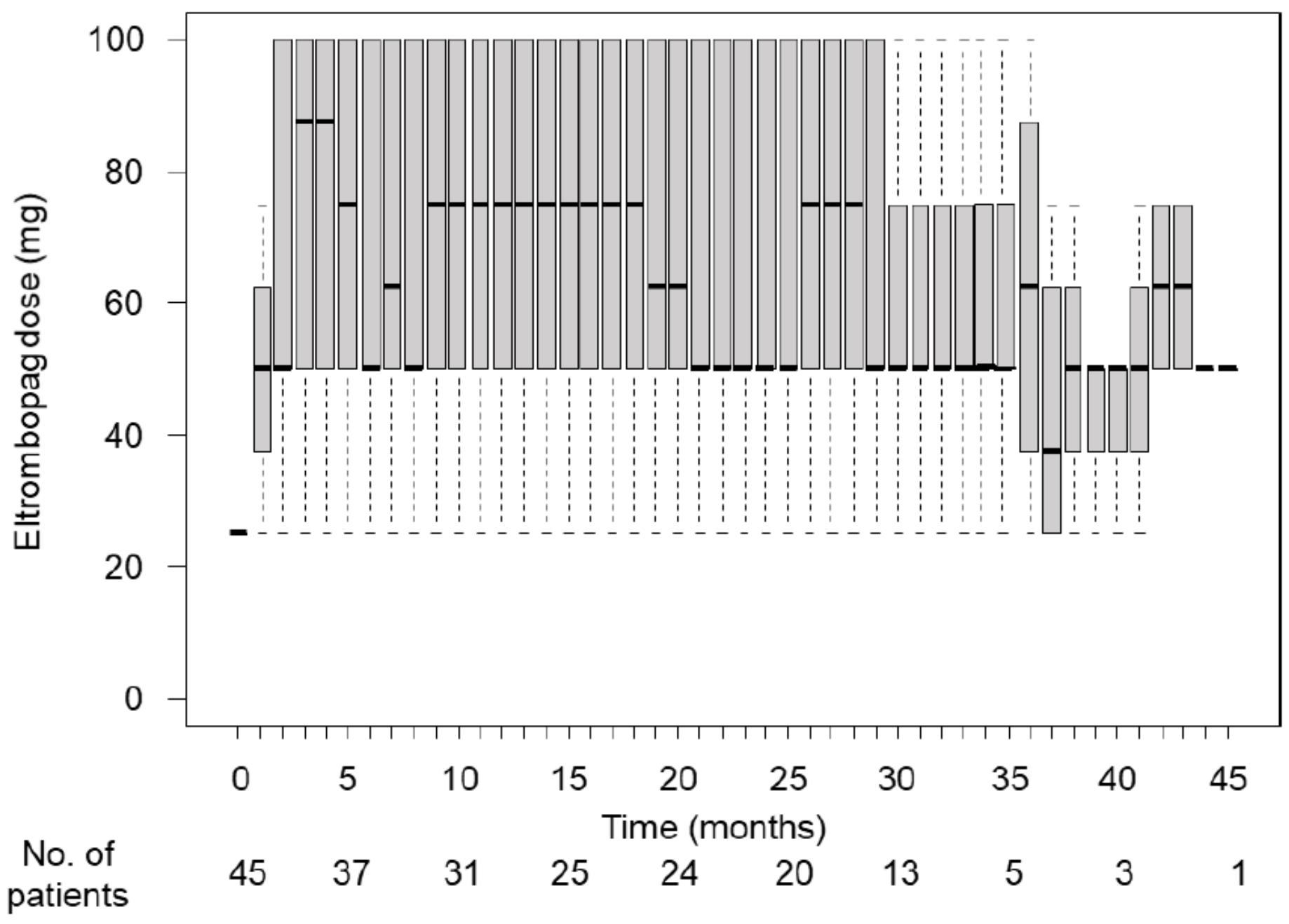

Figure 1

Eltrombopag dose over time. The horizontal line in the center of each box indicates the median; the upper and lower edges of the box indicate the 25th and 75th percentiles, respectively; and the bars indicate the range from the 5th to the 95th percentile. 

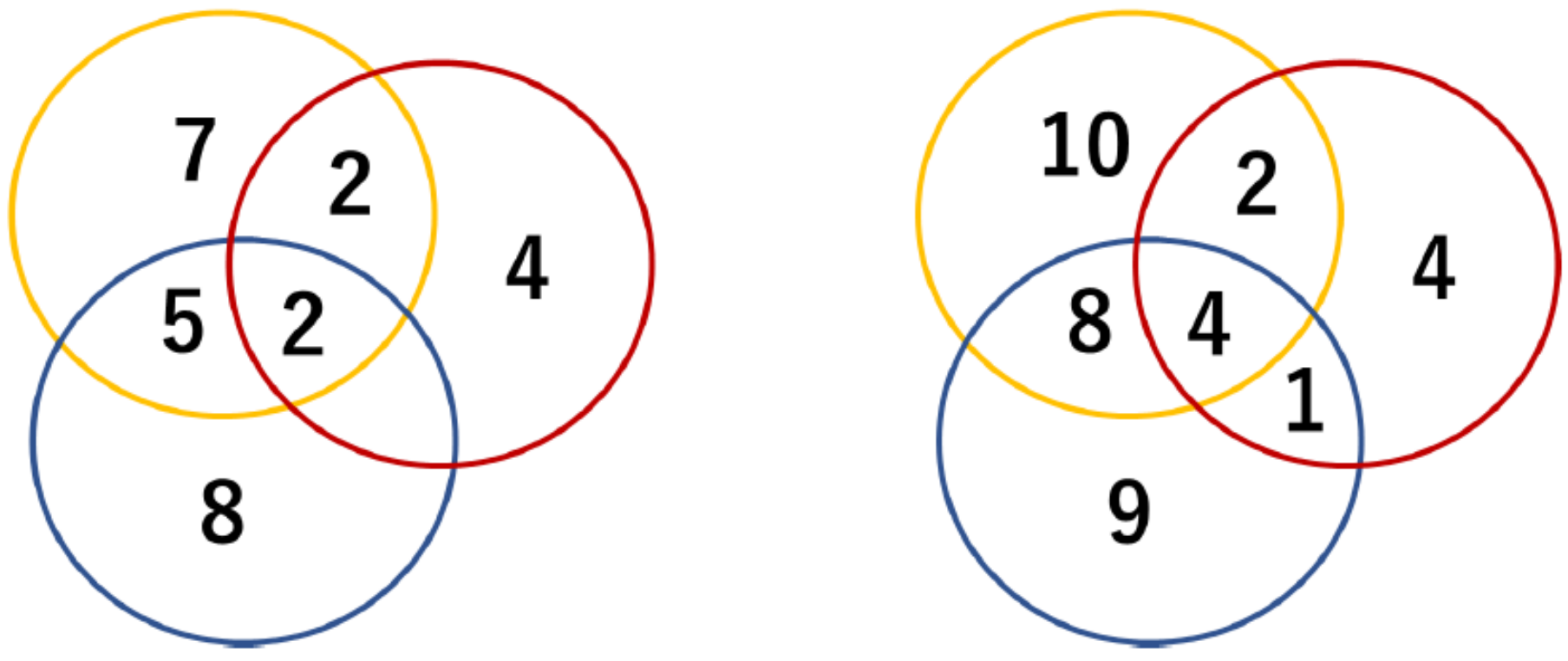

Figure 2

Response to TPO-RA by lineage. The Venn diagrams show the number of patients that had responses in each lineage at six months (A) and the best response during the entire follow-up period (B). The yellow, red, and blue circles indicate platelet, erythroid, and neutrophil responses, respectively.
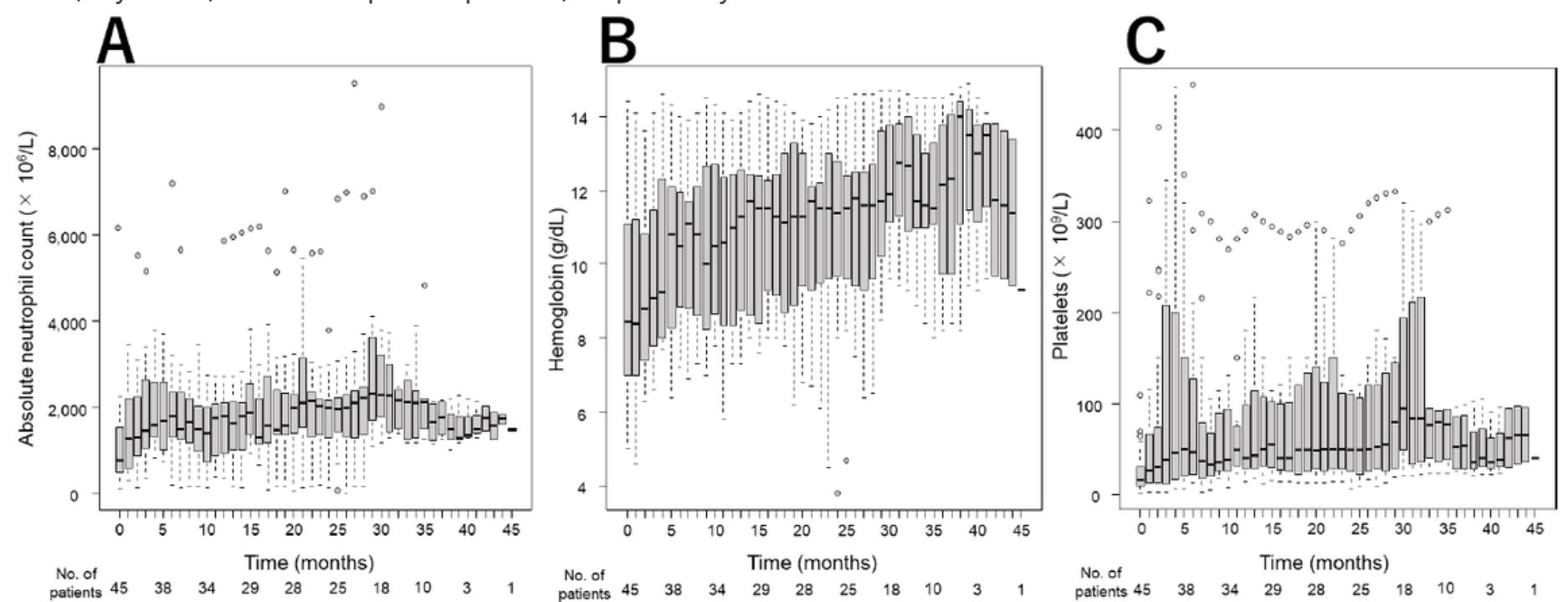

Figure 3

Response to TPO-RA treatment over time by lineage: neutrophil response (A), erythroid response (B), and platelet response (C). The horizontal line in the center of each box indicates the median; the upper and lower edges of each box indicate the 25th and 75th percentiles, respectively; and the bars indicate the range from the 5th to the 95th percentile. 